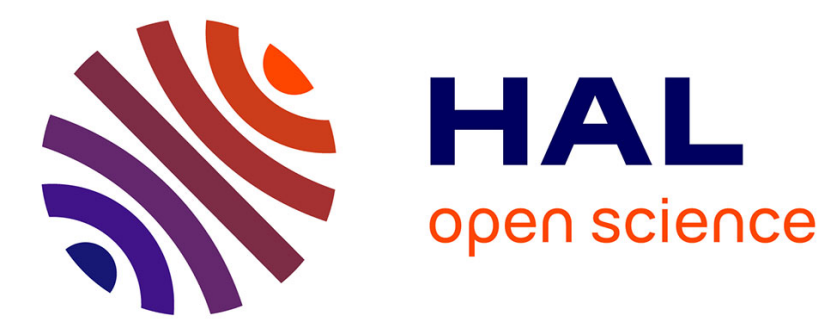

\title{
Performance scalability of the JXTA P2P framework
}

Gabriel Antoniu, Loïc Cudennec, Mike Duigou, Mathieu Jan

\section{To cite this version:}

Gabriel Antoniu, Loïc Cudennec, Mike Duigou, Mathieu Jan. Performance scalability of the JXTA P2P framework. [Research Report] RR-6064, 2006, pp.24. inria-00119916v3

\section{HAL Id: inria-00119916 https://hal.inria.fr/inria-00119916v3}

Submitted on 14 Dec 2006 (v3), last revised 20 Dec 2007 (v5)

HAL is a multi-disciplinary open access archive for the deposit and dissemination of scientific research documents, whether they are published or not. The documents may come from teaching and research institutions in France or abroad, or from public or private research centers.
L'archive ouverte pluridisciplinaire HAL, est destinée au dépôt et à la diffusion de documents scientifiques de niveau recherche, publiés ou non, émanant des établissements d'enseignement et de recherche français ou étrangers, des laboratoires publics ou privés. 


\section{Performance scalability of the JXTA P2P framework}

Gabriel Antoniu, Loic Cudennec, Mike Duigou, Mathieu Jan

\section{$\mathbf{N}^{\circ} \mathbf{1}$}

December 2006

Thème NUM

\section{apport}

de recherche 



\title{
Performance scalability of the JXTA P2P framework
}

\author{
Gabriel Antoniu*, Loic Cudennec*, Mike Duigou ${ }^{\dagger}$, Mathieu Jan* \\ Thème NUM — Systèmes numériques \\ Projets Paris
}

Rapport de recherche $\mathrm{n}^{\circ} 1$ - December 2006 - 21 pages

\begin{abstract}
Features of the P2P model, such as scalability and volatility tolerance, have motivated its use in distributed systems. Several generic P2P libraries have been proposed for building distributed applications. However, very few experimental evaluations of these frameworks have been conducted, especially at large scales. Such experimental analyses are important, since they can help system designers to optimize $\mathrm{P} 2 \mathrm{P}$ protocols and better understand the benefits of the P2P model. This is particularly important when the P2P model is applied to special use cases, such as grid computing. This paper focuses on the scalability of two main protocols proposed by the JXTA P2P platform. First, we provide a detailed description of the underlying mechanisms used by JXTA to manage its overlay and propagate messages over it: the rendezvous protocol. Second, we describe the discovery protocol used to find resources inside a JXTA network. We then report a detailed, largescale, multi-site experimental evaluation of these protocols, using the nine clusters of the French Grid' 5000 testbed.
\end{abstract}

Key-words: peer-to-peer, large scale, experimentation, peerview, discovery

This work has been supported by a Sun Microsystems - INRIA scientifi c collaboration contract (20052008) and by the Regional Council of Brittany, France.

* IRISA/INRIA, Campus de Beaulieu, 35042 Rennes, \{Gabriel.Antoniu\} \{Loic.Cudennec\} \{Mathieu.Jan\} @irisa.fr

$\dagger$ Project JXTA, Sun Microsystems, Santa Clara, CA, U.S.A, \{Mike.Duigou\} @ sun.com 


\section{Evaluation du passage à l'échelle de la plate-forme P2P JXTA}

Résumé : Les propriétés du modèle pair-à-pair (P2P), comme le passage à l'échelle et la tolérance à la volatilité, ont largement motivé son utilisation dans les systèmes distribués. Plusieurs librairies P2P génériques ont été proposées pour construire des applications distribuées. Cependant, très peu d'évaluations expérimentales de ces infrastructures ont été conduites à large échelle. Ce type d'expérimentation est pourtant important pour aider les concepteurs de systèmes $\mathrm{P} 2 \mathrm{P}$ à optimiser leurs protocoles et ainsi mieux appréhender les bénéfices du modèle $\mathrm{P} 2 \mathrm{P}$. Ceci est particulièrement vrai dans le cas d'utilisation du modèle P2P dans le contexte du calcul sur grille. Ce papier s'intéresse à l'aptitude du passage à l'échelle de deux protocoles proposés par la plate-forme P2P JXTA. Dans un premier temps, nous proposons une description détaillée des mécanismes utilisés par JXTA pour constituer son réseau logique et y propager des messages : le protocole de rendez-vous. Dans un second temps, nous décrivons le protocole de découverte des ressources. Enfin, nous présentons une évaluation expérimentale détaillée à grande échelle de ces protocoles en utilisant les neuf sites de la grille expérimentale française Grid'5000.

Mots-clés : pair-à-pair, large échelle, expérimentation, vue locale, découverte 


\section{Introduction}

Programming distributed applications has always been a difficult task. This difficulty is further increased when systems target a large scale (i.e. millions of users). While trying to address this problem, the Peer-to-Peer (P2P) approach is receiving a growing interest thanks to its properties, primarily scalability and volatility tolerance. Consequently, by using a P2P model, applications can hope to offer higher scalability and availability despite dynamic changes in the underlying physical infrastructure. The P2P model was initially used for large-scale applications over Internet (such as file sharing, instant messaging, etc.), but has also been found attractive in the field of distributed scientific simulation on grid infrastructures. As an example, it can typically be used in connection with grid resource management middleware [9,23].

Recently, a number of P2P libraries (e.g. Bamboo [28], FreePastry [29], JXTA [27], etc.) providing basic support for $\mathrm{P} 2 \mathrm{P}$ interaction (for example discovery mechanisms) have been made available. Such libraries are intended to serve as generic building blocks for higher-level P2P services and applications. Among these P2P libraries, the JXTA framework is emerging as a de facto standard for building services or applications in the industrial world [30]. It is also used in various research projects, $[1,2,21]$ to cite a few (see [31] for a more detailed list).

However, before using such generic layers, it is important to analyze their suitability with respect to the requirements of the target P2P service or application. Most published papers introducing these libraries give the cost of basic operations (e.g. routing and discovery) through complexity analyses and simulations. These theoretical evaluations are certainly necessary, but they clearly are only a preliminary step. To fully understand the behavior of the proposed P2P libraries, experimental evaluations on existing distributed testbeds are unavoidable. Such practical evaluations make it possible to better tune the proposed algorithms, depending for instance on the testbed scale or on the underlying network topology targeted by applications. For instance, the current convergence of grid computing and $\mathrm{P} 2 \mathrm{P}$ computing [10] call for precise requirements and guarantees to be defined for P2P algorithms. This makes it possible to better understand the benefits of the $\mathrm{P} 2 \mathrm{P}$ model in such particular use cases.

In this work, we focus on the performance of the JXTA protocols. JXTA is an opensource initiative, sparked by Sun Microsystems. It was founded in order to develop a set of standard open protocols for P2P network applications. To the best of our knowledge, it is the most advanced framework currently available for building services and applications based on the P2P model. In its 2.0 version, JXTA consists of a specification of six language- and platform-independent, XML-based protocols [32] that provide basic services common to most $\mathrm{P} 2 \mathrm{P}$ applications, such as peer group organization, resource discovery, and inter-peer

$\operatorname{RR} n^{\circ} 1$ 
communication. A more detailed overview of JXTA can be found in [27]. In this paper, we focus on the performance analysis of the so-called Loosely-Consistent Distributed Hash Table (LC-DHT) [26] at a large scale. This mechanism has been introduced in JXTA 2.0 for resource discovery.

In order to evaluate the cost of this LC-DHT, we benchmark the discovery protocol from an application point of view. However, we also evaluate the underlying algorithms used by the LC-DHT, i.e. the rendezvous protocol, used to organize the JXTA overlay and propagate queries. We perform multi-site tests over the Grid'5000 testbed [6], an experimental grid platform consisting of 9 sites geographically distributed in France. Grid' 5000 aims at gathering a total of 5,000 CPUs in the near future. We vary parameters of these algorithms: the overlay size and some parameters controlling the algorithm behavior. We also experiment two overlay topologies: chains and trees. These metrics are applied on the $\mathrm{C}$ reference implementation of the JXTA specifications, known as JXTA-C [25].

The remainder of the paper is organized as follows. Section 2 introduces related work: we discuss some existing performance evaluations of DHTs as well as of JXTA. Section 3 provides an overview of JXTA protocols stack, with a focus on protocols involved in the management of the LC-DHT evaluated in our experiments. Section 4 presents and discusses scalability experiments for the rendezvous protocol and for the discovery protocol. Finally, Section 5 concludes the paper and suggests directions for further research.

\section{Related work}

Papers introducing DHTs such as Pastry [19], have evaluated benefits of this approach, but mainly through theoretical analyses and simulations. The most common used metric to evaluate DHT is the number of hops required to look for a resource in a given overlay. Because of the use of simulations, past evaluations usually assume a static network. More recently, the impact of realistic conditions, such as for instance churns, on the performance of the look up operation have the subject of many papers [16, 18,22]. Such work are sometimes based on theoretical analyses [17], but more usually on laws modeling the session length of a peer $[16,18]$ as well as on traces of existing deployed systems [22]. However, note that traces are subject to inaccuracies: only a subset of the network has been explored in a time-constrained period and lookup patterns might therefore not be representative. Largescale experimental evaluations of DHTs in controlled environments are therefore required to compare any proposed improvements in a fair manner as well as to report performances of DHTs using traditional network metrics, such as latency, bandwidth, etc. To the best of our knowledge, only [8] provides such experimental evaluations, using 425 peers over 
150 nodes of the PlanetLab [7] testbed and takes into account underlying network characteristics in the used metrics.

In the JXTA field, various implementations of the JXTA specification have been evaluated $[11,12,20]$ and compared to other systems $[5,14]$ (especially as regards JXTA-J2SE). However most of these evaluations were performed at application-level, without any analysis of the internal behavior of JXTA's protocols. Such an analysis has recently been provided for the performance of JXTA's communication layers $[3,4]$. The goal of this paper is to bring a similar contribution by analyzing and evaluating JXTA's LC-DHT mechanism, on which relies JXTA's protocols for resource publishing and discovery.

The LC-DHT has been introduced in JXTA-J2SE and JXTA-C starting from versions 2.0 and 2.2 respectively. The approach has been compared to a classical DHT-based approach in [24], by modifying JXTA's discovery protocol. However, no performance evaluation was reported. In [13] authors compare the LC-DHT approach to a centralized or flooding approach (which was the strategy used by JXTA 1.0), with respect to memory usage, reliability and query response time for different configurations of a JXTA virtual network. Benchmarks were performed against JXTA-J2SE. However, no benchmarks have been performed at a large-scale, as the experimental configurations used up to 32 peers only, based on a LC-DHT distributed on 4 peers. One of the main goals of this paper is precisely to test the scalability of the LC-DHT approach, using much larger configurations.

\section{Description of JXTA}

\subsection{General overview}

JXTA relies on a set of basic concepts: a "peer" is an entity able to communicate by exchanging messages; a "peer group" (also called an overlay in the remainder of this paper) is a set of peers with a common interest, and providing common services; an "advertisement" is an XML document describing a resource. JXTA specifies a set of language- and platformindependent XML-based protocols [32]. These protocols are used inside each peer group to provide a rich set of building blocks (called services) for the management of peer-to-peer systems: resource discovery, overlay self-configuration, peer-to-peer communication, etc.

Figure 1 shows the stack of protocols defined by JXTA. Above the physical transport protocols, the endpoint routing protocol (ERP) is used to find available routes from a source peer to a destination peer. The rendezvous protocol is responsible for message distribution and topology management. On top of the rendezvous protocol, JXTA uses a standardized query/response protocol: the resolver protocol. It provides a generic, topology-independent query/response interface which other higher-level services may use to invoke various P2P

$\operatorname{RR} n^{\circ} 1$ 


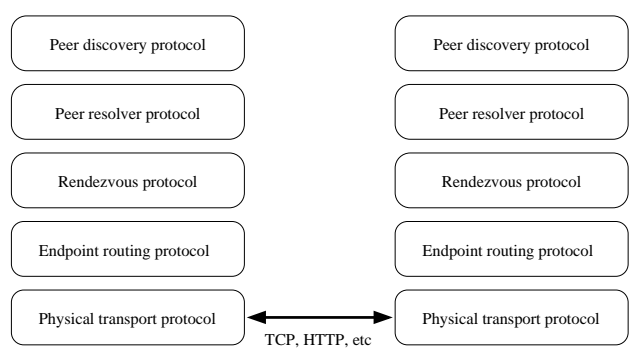

Figure 1: Partial view of JXTA protocol stack as defined by the specification.

operations. For instance, the discovery protocol defines one such service, which provides a specific API for publishing and discovery, which is implemented using the resolver protocol. The discovery protocol is used to publish and to find available resources within a JXTA overlay. It relies on the use of resource advertisements, whose life duration can controlled via the discovery API. Discovery resource announcements and queries are forwarded by the underlying resolver protocol to the appropriate rendezvous peers.

In this paper, we focus on the rendezvous protocol and on the discovery protocol. Before introducing in more details these two protocols, let us stress that, for each protocol, JXTA only defines the syntax for queries and responses; it does not specify the behavior of algorithms used by implementations willing to be compliant with the protocol specification. For instance, various message routing approaches can be used by JXTA implementations, making JXTA the perfect framework for testing topology-based routing algorithms. Moreover, the specification does not define the structure of a JXTA-based overlay. In current implementations (JXTA-C or JXTA-J2SE), a JXTA overlay is a structured network based on the use of mainly two peer types: super-peers, commonly rendezvous peers, and regular peers, called edge peers. Each edge peer is attached to a rendezvous peer. Queries and responses are forwarded among the super-peers using a Loosely-Consistent Distributed Hash Table (LC-DHT), whose behavior is detailed in Section 3.3. However, note that alternate implementations of JXTA may provide different types of overlays.

Let $\mathbf{S}$ be a JXTA overlay, composed of $r$ rendezvous peers and $e$ edge peers. If rendezvous peers are noted $R_{i}$, and edge peers are noted $E_{j}$, then $\mathbf{S}$ is defined as follows:

$$
\mathbf{S}=\left\{R_{i}, i=1 . . r\right\} \cup\left\{E_{j}, j=1 . . e\right\}
$$




\subsection{The peerview protocol: managing the overlay}

As stated by the JXTA specifications, the rendezvous protocol is divided into three subprotocols:

1. the peerview protocol, used by rendezvous peers to organize themselves by synchronizing their views of each other;

2. the rendezvous lease protocol, used by edge peers to subscribe to the reception of messages propagated by the rendezvous peers;

3. the rendezvous propagation protocol, which enables peers to manage the propagation of individual messages within a group.

In this paper, we focus on the first protocol: the peerview protocol. This protocol allows rendezvous peers to work together to form a so-called global peerview: an ordered list (by peer ID) of peers currently acting as rendezvous peers within a given group. This list is used for route messages within that group. Each rendezvous peer maintains a local version of the list which represents its view of the global peerview. Let $g$ be the size of the global peerview for a given peer group $\mathrm{S}$, and $l_{i}$ the size of local peerview of rendezvous peer $R_{i}$. Let $P V_{R_{i}}$ be the local peerview of rendezvous peer $R_{i}$ and $\mathbf{T}$ the set of values that can take the time during a given execution of a JXTA application. The goal of the peerview protocol is to form and keep the individual local peerviews consistent across all rendezvous peers participating in a given group. This can be translated in the following property:

$$
\exists t_{1} \in \mathbf{T}, \forall t_{2} \in \mathbf{T}, t_{2}>t_{1}, \forall R_{i} \in \mathbf{S}: l_{i}=g
$$

We shall see in Section 3.3 that this consistency is required for an optimal query propagation over a JXTA overlay.

To achieve this goal, rendezvous peers periodically probe other members of the peerview. Algorithm 1 shows the main steps of this process used by JXTA-C for this purpose. The algorithm is run by every rendezvous peer of $\mathbf{S}$. The elapsed time between two iterations of the algorithm is controlled by the PEERVIEW_INTERVAL constant (set by default to 30 seconds). Note that the algorithm used by JXTA-J2SE is slightly different, but the variation in behavior does not appear to be significant. Apart from the peer running the algorithm, two other peers are mainly involved: 1) the rendezvous peer whose peer ID immediately preceeds the local peer ID in the sorted list of peer IDs, noted as lower_rdv in the algorithm and 2) the rendezvous peer whose peer ID immediately follows the local peer ID in the same list, noted as upper_rdv in the algorithm. Theses two rendezvous peers, if present (peers at each end of the sorted list will have only one peer to probe), are

$\operatorname{RR} n^{\circ} 1$ 


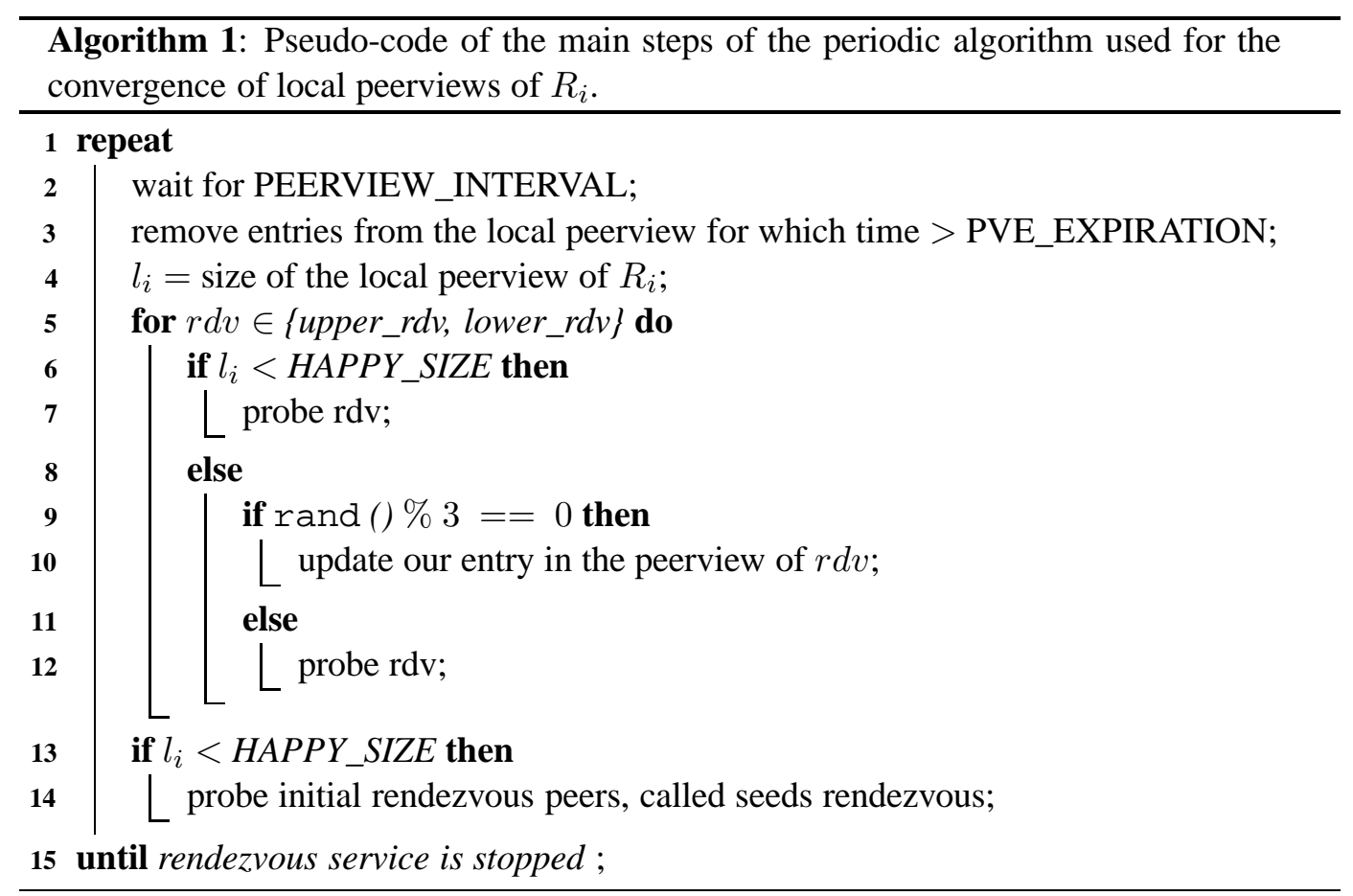


more actively probed if the size of the peerview has not reached a configurable minimum threshold: HAPPY_SIZE (by default set to 4). A probe is a peerview message that contains a rendezvous advertisement describing the sender (let us call this peer $A$ ). In response to a probe, the receiver (also a rendezvous peer, let us call it $B$ ) returns its own rendezvous advertisement. In a separate message, peer $B$ will also return a randomly chosen rendezvous advertisement for another rendezvous peer in his list $(C)$. This second message is known as a referral response. This way, the initiator of the probe $(A)$ may learn about a new rendezvous peer. However, before adding this new rendezvous advertisement in its local peerview, peer $A$ will probe peer $C$, which will also send a referral response publishing the identity of yet another rendezvous peer $(D)$, and so on. The default lifetime of rendezvous advertisements in the peerview is controlled via a constant (PVE_EXP IRATION, by default set to 20 minutes). This tunable constant is used at line 3 of Algorithm 1 to remove expired peerview entries.

\subsection{The discovery protocol: publishing and discovering resources}

An overview of the LC-DHT algorithm. The LC-DHT algorithm (for Loosely-Consistent Distributed Hash Table) defines a mechanism for publishing and discovering resources within a JXTA overlay. Each resource made available is described by an advertisement. Each type of advertisement defines a set of attributes by which instances of the advertisement are indexed. Peers maintain and publish attribute tables for their advertisements. An attribute table consists of tuples (index attribute, value), each of which is associated to a life duration and to the identity of the publishing peer. These attribute tables are published by the edge peers to their associated rendezvous peers. Rendezvous peers which receive such publication requests keep a copy of the tuples and then replicate them across the entire network of rendezvous by applying a hash function on each tuple. The hash function (detailed below) shows that the mapping of indexes to the appropriate replica rendezvous peer is based on the use of the local peerview (we remind the reader that $l_{i}$ is the size of the local peerview):

Function ReplicaPeer (tuple) applied by peer $R_{i}$ member of $\mathbf{S}$ to find the replica peer for a given advertisement.

1 hash = SHA-1 (tuple);

2 pos $=$ floor $\left(\right.$ hash $\left.* \frac{l_{i}}{M A X_{-} H A S H}\right)$;

3 Return peerview entry at position pos;

$\operatorname{RR} n^{\circ} 1$ 


\begin{tabular}{|l|l|l|l|l|l|l|}
\hline Peerview entry & 0 & 1 & 2 & 3 & 4 & 5 \\
\hline Peer ID $\left(R_{i}\right)$ & $006\left(R_{1}\right)$ & $020\left(R_{2}\right)$ & $036\left(R_{3}\right)$ & $050\left(R_{4}\right)$ & $088\left(R_{5}\right)$ & $180\left(R_{6}\right)$ \\
\hline
\end{tabular}

Table 1: Local peerview used by each $R_{i}$ member of $\mathbf{S}$ for both publish and lookup operations of tuple (116, E1).

The hash is actually applied on a string obtained by concatenating the type of the advertisement, the name of the attribute used for indexing and its value. The maximum value the hash function of the LC-DHT can return is noted MAX_HASH. A replica peer of an advertisement is a rendezvous peer designated by the LC-DHT algorithm as that responsible for that advertisement.

Let us take an example to illustrate both publish and lookup operations for a resource (with $\mathbf{S}=\left\{R_{i}, i=1 . .6\right\} \cup\left\{E_{j}, j=1 . .2\right\}$ ). In our example the resource is a peer represented by a peer advertisement Adv (so the peer type is Peer); let us assume that the index attribute is Name and its associated value is Test. The hash function will then be applied to the string: "PeerNameTest". Let us assume that the output hash value is 116, that MAX_HASH equals 200 and that the property (2) is satisfied $\left(l_{i}=g=6\right)$. Consequently, the Table 1 shows local peerviews of each $R_{i}$ member of $\mathbf{S}$.

Publishing. The left side of Figure 2 shows the main steps for publishing this advertisement, which are described below.

1. The tuple is first sent by the edge peer $E_{1}$ to its associated rendezvous peer $R_{1}$, which can compute the appropriate replica peer for the advertisement. According to the replica peer function (position $=\left\lfloor\frac{116 * 6}{200}\right\rfloor=3$ ), the tuple $(116$, E1) will get replicated onto the third peer in $R_{1}$ 's peerview, which is rendezvous peer $R_{4}$ (see Table 1$)$. The tuple $(116, \mathrm{E} 1)$ is also stored by $R_{1}$ to increase its availability for edge peers connected to the same rendezvous peer.

2. The tuple $(116, \mathrm{E} 1)$ is therefore sent on the rendezvous peer $R_{4}$.

Discovery. The goal of the peer discovery protocol is to find resources within the group. It makes use of the same tuples described above. Edge peers specify queries by indicating index attributes and desired values for those attributes. This is expressed as a discovery protocol message, which is propagated over a JXTA overlay according to the following steps. 

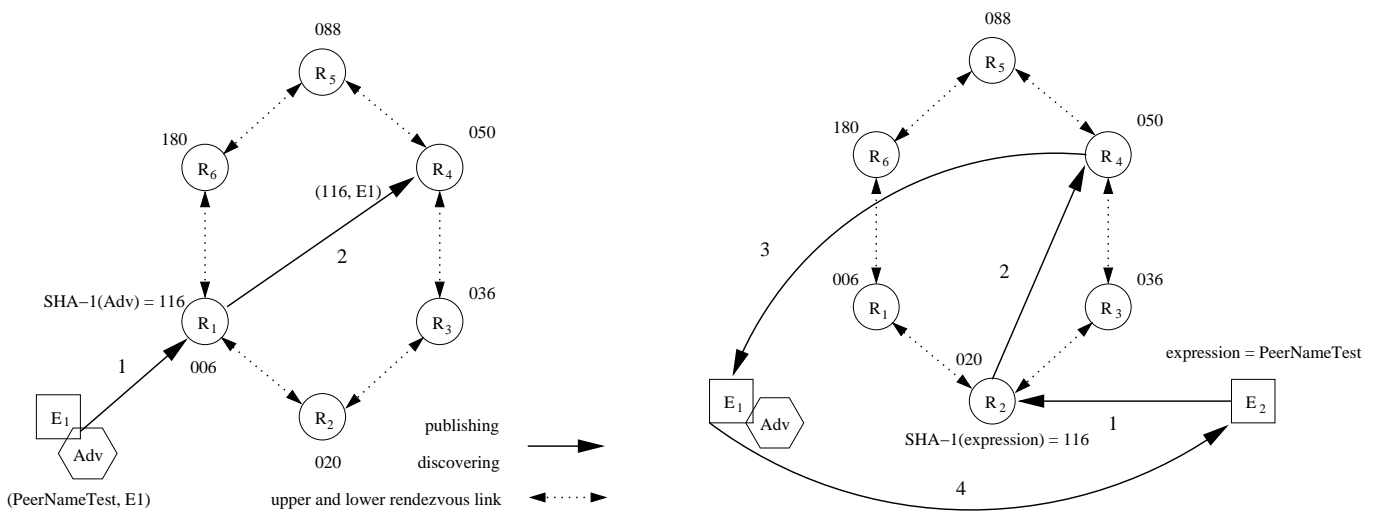

Figure 2: Main steps for publishing (left) and looking up (right) a resource advertisement over the network of rendezvous peers. In this example, the advertisement has a hash value of 116 .

1. The message is first forwarded by the edge peer to its rendezvous peer via the resolver protocol (for rendezvous peers this step is not necessary as they act as their own rendezvous). In our example, edge peer $E_{2}$ is looking up for Adv: the discovery request is sent to $R_{2}$, which is $E_{2}$ 's rendezvous peer.

2. Rendezvous peer $R_{2}$ first checks if it has a local hash value of 116. In that case, $R_{2}$ can directly forwards the query to $E_{1}$ and the lookup process continues at step 4 . If no local matching hash value if found, $R_{2}$ applies the same hash function as used for publication upon the query expression elements. Consequently, the query is forwarded to the same resulting replica peer: $R_{4}$.

3. As $R_{4}$ is indeed the correct replica peer for this query, the query is forwarded to the edge peer that has published the Adv advertisement: $E_{1}$.

4. Finally, edge peer $E_{1}$ sends its advertisement to the requesting edge peer $\left(E_{2}\right)$.

Link with the peerview protocol. When publishing or looking up for advertisements, local peerviews of rendezvous peers are used to forward messages to replica peers. However, if rendezvous peers have different local peerviews they will route messages to different destination rendezvous peers: this is due to the fact that the replica peer is computed based on the rank of the rendezvous peers in the local peerviews. If local peerviews differ on the 
rendezvous peer performing the publication and on the rendezvous peer in charge of performing the corresponding discovery query, the replica peer computed at publication will not match the one computed during the discovery process. However, upon failing to find a resource on a replica peer, a backup mechanism is used: the query will be forwarded to the upper and lower rendezvous peers, which may store the resource. The query is said to walk the whole peerview in both directions: towards upper values in the hash space starting from the upper_rdv rendezvous peer, and towards lower values in the hash space starting from the lower_rdv rendezvous peer.

Complexity. On an overlay gathering $n$ nodes, classical DHTs have a complexity in $O(\log n)$ for publishing resources ${ }^{1}$, whereas LC-DHT have a complexity in $O(1)$ (2 messages in the worst case). However for discovery, a LC-DHT based approach does not offer the same $O(\log n)$ query guarantee that other DHT systems provide. Current implementations of JXTA provide a $O(r)$ query guarantee, where $r$ is the number of rendezvous peers. The worst case corresponds to the use of the walk mechanism upon failure of the hash-based discovery (due to high churn), more precisely when the rendezvous peer responsible for the searched advertisement is diametrically opposed in the peerview of the rendezvous peer computed by the hash function. Therefore the peerview must be walked and the number of hops used is then in $O(r)$. In practice, with the help of advertisement replication, this number of hops is much less than $r$. Finally, note that if local peerviews of all rendezvous peers of a given group satisfy the property (2), the complexity is only in $O(1)$ (actually 4 messages in the worst case, as illustrated by the right side of Figure 2).

The LC-DHT approach avoids the expensive traffic (and, often more importantly, latency overhead) required by classical DHTs to maintain consistency. On the other hand, if rendezvous peers are volatile, peerviews may change, and computed replica peers may not be correct, as it would be theoretically the case with classical DHTs. However, studies of these classical DHTs showed that their maintenance mechanisms are unable to cope with churns of a few tens of minutes [18], which most peers of existing systems follow according to the same paper. Note that JXTA edge peers periodically push tuples of updated or new indexes to their rendezvous peers (by default every 30 seconds). However, this is only done if advertisements have changed or have been explicitly republished by applications. Consequently, this may lead to the computation of new replica peers, to allow future discovery requests to complete correctly. On the other hand, edge peers also publish their tuples whenever they connect to a new rendezvous peer.

The drawback of this LC-DHT approach is therefore that it is possible to deliver incomplete results in the case of highly inconsistent peerviews. This explains the name given to

\footnotetext{
${ }^{1}$ With respect to the number of required hops.
} 
this approach: loosely-consistent $D H T$, whose aim is to cope with highly-dynamic peer to peer networks.

\section{Scalability evaluation of JXTA-C protocols}

Experimental setup. Nodes used for the experiments mainly consist of machines using dual or quadri 2.2 GHz AMD Opteron, dual $900 \mathrm{MHz}$ Intel Itanium2, outfitted with up to 4 GB of RAM each, and running a 2.6 version Linux kernel; the hardware network layer used is a Giga Ethernet $(1 \mathrm{~Gb} / \mathrm{s})$ network. All 9 sites of the Grid' 5000 testbed were used: Bordeaux, Grenoble, Lille, Lyon, Nancy, Orsay, Rennes, Sophia and Toulouse. Tests were executed using JXTA-C version 2.3 (released the 15th of December 2005), used and configured to use TCP as the underlying transport protocol. JXTA-C benchmarks are compiled using gcc 4.0 with the -02 level of optimization. Finally, for the deployment of JXTA overlays, we used the generic deployment tool ADAGE [15]. We indeed developed a JXTA plug-in for ADAGE so that overlays can be described in a concise manner, and generation of configuration files for JXTA automated. We performed two different kind of tests.

\subsection{Evaluation of the peerview protocol}

Benchmark description. The goal of this benchmark is to measure the time it takes for the peerview protocol to make the LC-DHT consistent across rendezvous peers, that is to say the time for the property (2) to be satisfied. Each time a rdv peer is added to/removed from the local peerview of a rendezvous peer, the elapsed time since the beginning of the test is logged, as well as the type of event. We performed benchmarks using an increasing number of rendezvous peers, with different logical topology (chain or tree) and for different values of the PVE_EXPIRATION constant. As in Section 3.1, we note $r$ the number of rendezvous peers in a peer group (noted $\mathbf{S}$ as introduced in the same Section) and $l$ the size of the local peerview of a rendezvous peer. Finally, note that in our benchmark $\mathbf{S}$ is only made of rendezvous peers $\left(\mathbf{S}=\left\{R_{i}, i=1 . . r\right\}\right)$.

Scalability of the peerview protocol. The left side of Figure 3 shows the evolution of $l$ according to $r$. Both chains ( $r$ equals to $10,45,50,80,160,580)$ and trees $(160,220,338)$ topologies have been tested, revealing this initial parameter has no significant influence on the peerview behavior. For a same experiment, the value $l$ of each rendezvous peer belonging to $\mathbf{S}$ evolves in the same way. When $r \geq 45$, the property (2) is not satisfied. Moreover, only three experiments ( $r$ equals to 10, 45 and 50) reach the maximal possible

$\operatorname{RR} n^{\circ} 1$ 

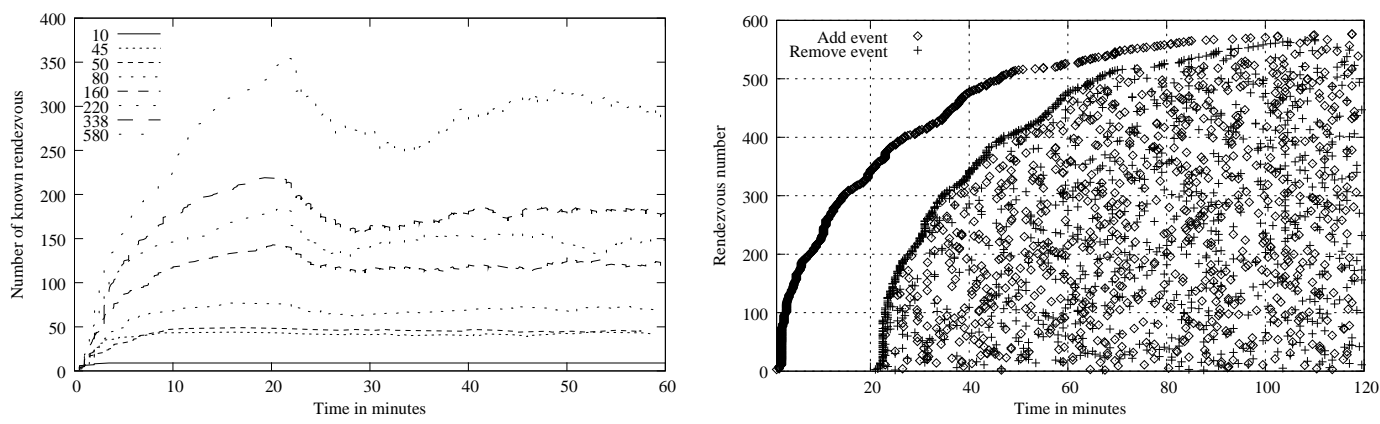

Figure 3: Evolution of the size $l$ of the peerview of a rendezvous peer according to the total number $r$ of rendezvous peers in the system (left). Distribution of add and remove events of rendezvous peers in the peerview of a rendezvous-peer (right).

value for $\mathbf{S}$. This maximal possible value, noted $m$, is equal to $r-1^{2}$. Finally, the experiment with $n=580$ enables to clearly distinguish three phases in the setup on the local peerview of a rendezvous peer.

1. An increase of $l$. This phase lasts as long as the lifetime duration of the advertisements of rendezvous peers (controlled by the constant PVE_EXPIRATION, see Section 3.2), which is 20 minutes for the given experiment.

2. A decrease of $l$. This phase starts at the time PVE_EXPIRATION. If after this time the rendezvous associated to its entry in the peerview is not probed by the algorithm of the peerview, the entry is removed from it.

3. A fluctuation of $l$ around a given value, depending of the value of $n$. In our case, $l$ is around 300. In the worst case, this phase starts at a time around twice the value of PVE_EXPIRATION in minutes (experiment with $r=580$ ).

The right side of Figure 3 shows the distribution of adding and removal events (respectively depicted by rhombus and cross) of rendezvous peers in the local peerview of a rendezvous peer (where $r=580$ ). More precisely, on the $y$ axis is shown the number of a given rendezvous peer ${ }^{3}$ of the experiment. Results show that almost all rendezvous peers are getting in touch with all other rendezvous peers. The maximal given number of the last

\footnotetext{
${ }^{2}$ Our measurement excludes the local rendezvous peer from the size of the peerview.

${ }^{3}$ For each new rendezvous peer added in the peerview, a number is given to the rendezvous peer starting from 1 .
} 
know rendezvous peer is indeed 577, that is to say 2 rendezvous peers were not discovered. This occurs 117 minutes after the beginning of the experiment. Two phases can also be observed for this curve:

1. Only adding events of rendezvous peers in the peerview occur. This phase lasts $\mathrm{PVE}$ EXP IRATION (in our experiment 20 minutes). It starts with a curve of adding events in local peerviews, as shown by the first curve, made of rhombus, on the right side of Figure 3.

2. Both adding and removal events of rendezvous peers in the peerview are observed. This phase starts at time PVE_EXPIRATION and lasts till the end of the experiment. It starts with a curve of removal events in local peerviews, as shown by the second curve, made of crosses, on the right side of Figure 3.

Figure 3 (right side) explains the third phase in the setup of the peerview of a rendezvous peer. The fluctuation of the value $m$ corresponds indeed to the adding and removal of entries in the peerview, which occur in this second and last phase (right side Figure 3). They indicate the incapacity of the peerview protocol to probe all the entries of the peerview, in a time shorter than the value of the constant PVE_EXPIRATION. Moreover, this figure also explains why the value of $m$ culminates in time to the value of the constant $\mathrm{PVE}_{\text {_ }}$ EXPIRATION. After this time, the curve of adding events counterbalances the curve of removal events, thereby explaining the phase 3 in the setup of a local peerview.

Discussion. This benchmark allows to characterize the behavior of the algorithm of the peerview protocol of JXTA-C. It tries to give a reliable answer to the following question: how many rendezvous peers can be deployed in a JXTA group? This question is frequently asked in the mailing lists of JXTA and until now answered without any proof. The peerview algorithm was believed to work well for values of $n$ as high as 100 or 150 . However, our evaluation shows that even for $n$ greater than 45 , the property (2), which expresses the goal of the peerview protocol, is not satisfied. Consequently, with parameters of the peerview protocol set to default values, a JXTA group made of 45 rendezvous peers is not able to optimally organize itself. Therefore, the most efficient routing of requests is not guaranteed, leading to an increase in the number of hops needed for a discovery request for example (see Section 3.3).

A solution is to modify the value of the constant PVE_EXPIRATION, as shown by the following experiment. The Figure 4 shows the evolution of the value of $m$ on a rendezvous peer (with $r=50$ ), according to two different values for the constant PVE_EXPIRATION. By changing this constant to a time greater than the duration of the experiment (60 minutes in our case), $l$ reaches its maximum possible value: $r-1$, which in our case is 49 . In

$\operatorname{RR} n^{\circ} 1$ 

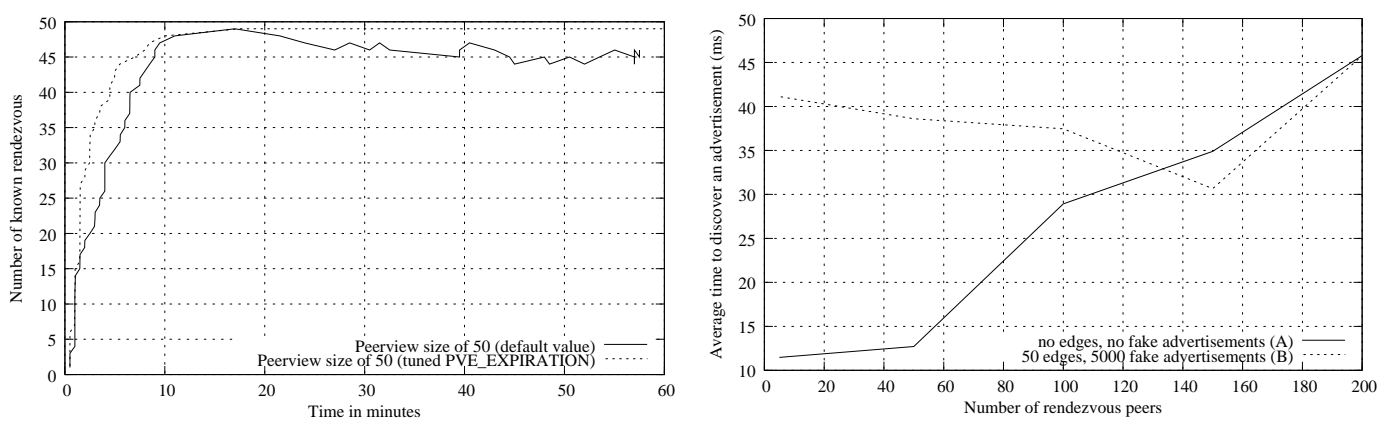

Figure 4: Evolution of the size $l$ of the peerview of a rendezvous peer, for a P2P system made of 50 rendezvous peers, according to the value of the parameter PVE_EXPIRATION (left). Time $t$ to discover an advertisement depending on the number of rendezvous peers $r$, edge peers and fake advertisements $f$ (right).

Property (2), $t_{1}$ is therefore equal to 17 minutes. Another solution to support a higher number of rendezvous peers is to decrease the interval of time between each iteration of the peerview algorithm loop (constant PEERVIEW_INTERVAL, see Section 3.2). Note that both parameters can be tuned at the same time.

In all cases, a compromise must be reached between freshness (and thereby reliability of information in the peerview) on one side and bandwidth consumption on the other side. The freshness of information decreases when the value of the constant PVE_EXP IRATION increases, whereas the bandwidth consumption increases whenever the value of the constant PEERVIEW_INTERVAL increases.

\subsection{Evaluation of the discovery protocol}

Benchmark description. The goal of this benchmark is to evaluate the time $t$ needed for an edge to retrieve an advertisement. In our setup, a network of $r$ rendezvous peers is deployed to route discovery messages. One edge (called publisher) connects to this network and publishes a specific advertisement that is then searched by another edge (called searcher). All measurements are calculated based on 100 consecutive queries, each of them followed by a flush of the local searcher cache, in order to avoid cache speedup. Publishing and searching jobs delay their execution time after that local peerviews of rendezvous peer entered in their phase 3 (see explanations of Figure 3). A first set of experiments involves a publisher, a searcher and an increasing number of rendezvous peers 
(configuration $A$ ). No edge peers are attached to rendezvous peers but the publisher and the searcher $\left(\mathbf{S}=\left\{R_{i}, i=1 . . r\right\} \cup\left\{E_{j}, j=1 . .2\right\}\right)$. The second set of experiments extends the first one by adding edge peers to some rendezvous peers (configuration $B$ ). More precisely, 50 edge peers will connect to 5 rendezvous peers amongst the $r$ available $\left(\mathbf{S}=\left\{R_{i}, i=1 . . r\right\} \cup\left\{E_{j}, j=1 . .52\right\}\right)$. Each peer of this class, called noisers, publish a specified number of random advertisements $f$, called fake advertisement, to its rendezvous peer. The goal of this second experiment is to stress the discovery protocol by running it in more realistic conditions, where applications publish multiple advertisements concurrently. This will allow us to measure the impact of concurrency on the discovery time.

Scalability of the discovery protocol. The right side of Figure 4 shows the average time $t$ needed to discover a given advertisement, according to the number of rendezvous peers $r$. For each value of $r$ we measure the discovery time with $(B)$ and without $(A)$ extra "noise" produced by 50 noiser edges (with $f=100$ ). The (A) curve shows that adding up to 50 rendezvous peers does not significantly increase the discovery time: $t$ remains around $12 \mathrm{~ms}$. From 50 to 200 rendezvous peers, the discovery time grows linearly. This is explained by the results explained above on the peerview protocol: when using such a large number of rendezvous peers, Property (2), which is related to the stabilization of the peerview, cannot be satisfied. Therefore, the local peerviews of rendezvous peers are not consistent, forcing the LC-DHT algorithm to walk the global peerview. This represents a linear cost with respect to the number of rendezvous peers $r$, as stated in the complexity paragraph of Section 3.3. The $(B)$ curve shows the impact of the "noise" on the discovery time, a total of 5,000 fake advertisements are published by the 50 noisers. The maximum overhead is measured for $r=5(30 \mathrm{~ms})$, i.e. when noisers are attached to each rendezvous peer of the network. Then, for values of $r$ up to 150, this overhead slightly decreases. This can be explained by the load balancing of publishing queries onto different replica peers, as we use the same number of edge peers $e$ (always equal to 50). From $r=150$ to $r=200$, publishing fake advertisements no longer influence the discovery time.

Discussion. As seen in Section 2, DHT algorithms are mainly benchmarked using the number of hops required to perform a lookup. In addition to such an analysis of the discovery protocol (see Section 3.3), we could measure the latency of discovery requests. Such results enable to tune applications based on this protocol depending on the network size $(r)$ and the number of published advertisements. Therefore, they help finding an answer to the following question: when should the application re-launch a discovery query?

$\operatorname{RR} n^{\circ} 1$ 


\section{Conclusion}

The interesting features of the $\mathrm{P} 2 \mathrm{P}$ model have made it attractive for both the academic and industrial world. Several generic P2P frameworks are now available to developers wishing to use this model for their applications. However, very few experimental evaluations of these P2P libraries have been reported, especially at large scales.

In this paper, we focus on a de facto standard of P2P programming: JXTA specifications. We provide a detailed analysis of the protocol used to manage a JXTA overlay, namely the peerview protocol. We also perform an analysis of the protocol used to lookup for resources: the discovery protocol. The theoretical behavior of both protocols is described and multi-site experimental tests are reported, using the French Grid'5000 testbed with various JXTA-C overlay configurations. The goal of these benchmarks is to answer a common and unanswered question on the JXTA mailing lists: how many rendezvous peers are supported by JXTA in a given group? Our results show that with default values for parameters of the peerview protocol, the goal of the algorithm is not achieved, even with as few as 45 rendezvous peers. However, parameter tuning makes it possible to reach larger configurations in terms of number of rendezvous peers. For the discovery protocol, we show that discovery time is rather smaller, provided that all rendezvous peers satisfy a given property. These results give developers a better view of the scalability of JXTA protocols.

Nevertheless, considering all the factors explored in this paper, this research is not an exhaustive evaluation of the scalability of JXTA protocols. In particular, no volatility was introduced during the experiments. For instance, it would be interesting to evaluate the behaviour of fall-back mechanism used for resource discovery under high volatility. Further experiments should also evaluate the mechanisms used by JXTA-C to address complex queries, such as range queries.

\section{References}

[1] Michele Amoretti, Gianni Conte, Monica Reggiani, and Francesco Zanichelli. Service Discovery in a Grid-based Peer-to-Peer Architecture. In International Workshop on e-Business and Model Based IT Systems Design, Saint Petersburg, Russia, April 2004.

[2] Gabriel Antoniu, Luc Bougé, and Mathieu Jan. JuxMem: An Adaptive Supportive Platform for Data Sharing on the Grid. Scalable Computing: Practice and Experience, 6(3):45-55, September 2005.

[3] Gabriel Antoniu, Philip Hatcher, Mathieu Jan, and David A. Noblet. Performance Evaluation of JXTA Communication Layers. In Proc. Workshop on Global and Peer-to-Peer Computing 
(GP2PC 2005), pages 251-258, Cardiff, UK, May 2005. Held in conjunction with the 5th IEEE/ACM Int. Symp. on Cluster Computing and the Grid (CCGrid 2005).

[4] Gabriel Antoniu, Mathieu Jan, and David A. Noblet. Enabling the P2P JXTA Platform for High-Performance Networking Grid Infrastructures. In Proc. of the first Intl. Conf. on High Performance Computing and Communications (HPCC '05), number 3726 in Lect. Notes in Comp. Science, pages 429-440, Sorrento, Italy, September 2005. Springer.

[5] Sébastien Baehni, Patrick Th. Eugster, and Rachid Guerraoui. OS Support for P2P Programming: a Case for TPS. In 22nd International Conference on Distributed Computing Systems (ICDCS '02), pages 355-362, Vienna, Austria, July 2002. IEEE Computer Society.

[6] Franck Cappello, Eddy Caron, Michel Dayde, Frederic Desprez, Emmanuel Jeannot, Yvon Jegou, Stephane Lanteri, Julien Leduc, Nouredine Melab, Guillaume Mornet, Raymond Namyst, Pascale Primet, and Olivier Richard. Grid'5000: A Large Scale, Reconfi gurable, Controlable and Monitorable Grid Platform. In Proceedings of the 6th IEEE/ACM International Workshop on Grid Computing (Grid '05), pages 99-106, Seattle, WA, USA, November 2005.

[7] Brent Chun, David Culler, Timothy Roscoe, Andy Bavier, Larry Peterson, Mike Wawrzoniak, and Mic Bowman. PlanetLab: An Overlay Testbed for Broad-Coverage Services. ACM SIGCOMM Computer Communication Review, 33(3):3-12, July 2003.

[8] Frank Dabek, Jinyang Li, Emil Sit, James Robertson, M. Frans Kaashoek, and Robert Morris. Designing a DHT for Low Latency and High Throughput. In Proc. of the 1st Symposium on Networked Systems Design and Implementation (NSDI 2004), pages 85-98, San Francisco, CA, USA, March 2004. USENIX.

[9] Niels Drost, Rob van Nieuwpoort, and Henri E. Bal. Simple Locality-Aware Co-allocation in Peer-to-Peer Supercomputing. In Proceedings of the 6th IEEE/ACM International Symposium on Cluster Computing and the Grid (CCGrid '06), May 2006.

[10] Ian Foster and Adriana Iamnitchi. On Death, Taxes, and the Convergence on Peer-to-Peer and Grid Computing. In 2nd International Workshop on Peer-to-Peer Systems (IPTPS '03), number 2735 in Lect. Notes in Comp. Science, Berkeley, CA, USA, February 2003. Springer.

[11] Emir Halepovic and Ralph Deters. The Cost of Using JXTA. In 3rd International Conference on Peer-to-Peer Computing (P2P '03), pages 160-167, Linköping, Sweden, September 2003. IEEE Computer Society.

[12] Emir Halepovic and Ralph Deters. JXTA Performance Study. In IEEE Pacific Rim Conference on Communications, Computers and Signal Processing (PACRIM '03), pages 149-154, Victoria, B.C., Canada, August 2003. IEEE Computer Society.

[13] Emir Halepovic, Ralph Deters, and Bernard Traversat. Performance Evaluation of JXTA Rendezvous. In International Symposium on Distributed Objects and Applications (DOA '04), pages 1125-1142, Agia Napa, Cyprus, October 2004. Springer.

$\mathrm{RR} \mathrm{n}^{\circ} 1$ 
[14] Markus Junginger and Yugyung Lee. The Multi-Ring Topology - High-Performance Group Communication in Peer-to-Peer Networks. In 2nd International Conference on Peer-to-Peer Computing ( $P 2 P^{\prime}$ '02), pages 49-56, Linköping, Sweden, September 2002. IEEE Computer Society.

[15] Sébastien Lacour, Christian Pérez, and Thierry Priol. Generic application description model: Toward automatic deployment of applications on computational grids. In 6th IEEE/ACM International Workshop on Grid Computing (Grid2005), Seattle, WA, USA, November 2005. Springer.

[16] Jinyang Li, Jeremy Stribling, Robert Morris, M. Frans Kaashoek, and Thomer M. Gil. A performance vs. cost framework for evaluating DHT design tradeoffs under churn. In Proc. 24th Conf. on the IEEE Computer and Communications Societies (INFOCOM 2005), pages 225-236. IEEE Computer Society, March 2005.

[17] David Liben-Nowell, Hari Balakrishnan, and David Karger. Analysis of the evolution of peerto-peer systems. In Proc. of the 21 th annual symposium on Principles of distributed computing (PODC '02), pages 233-242, Monterey, CA, USA, 2002. ACM Press.

[18] Sean Rhea, Dennis Geels, Timothy Roscoe, and John Kubiatowicz. Handling Churn in DHT. Technical Report UCB/CSD-03-1299, Univeristy of California, Computer Science Division (EECS), Berkeley, CA, USA, December 2003.

[19] Antony I. T. Rowstron and Peter Druschel. Pastry: Scalable, Decentralized Object Location, and Routing for Large-Scale Peer-to-Peer Systems. In Proceedings of the 18th IFIP/ACM International Conference on Distributed Systems Platforms (Middleware 2001), volume 2218 of Lecture Notes in Computer Science, pages 329-250, Heidelberg, Germany, November 2001. Springer.

[20] Jean-Marc Seigneur, Gregory Biegel, and Christian Damsgaard Jensen. P2P with JXTA-Java pipes. In 2nd international Conference on Principles and Practice of Programming in Java (PPPJ '03), pages 207-212, Kilkenny City, Ireland, 2003. Computer Science Press, Inc.

[21] Kazuyuki Shudo, Yoshio Tanaka, and Satoshi Sekiguchi. P3: P2P-based Middleware Enabling Transfer and Aggregation of Computational Resources. In Proc. Workshop on Global and Peer-to-Peer Computing (GP2PC 2005), pages 259-266, Cardiff, UK, May 2005. Held in conjunction with the 5th IEEE/ACM Int. Symp. on Cluster Computing and the Grid (CCGrid 2005).

[22] Daniel Stutzbach and Reza Rejaie. Improving lookup performance over a widely-deployed DHT. In Proc. 25th Conf. on Computer Communications (INFOCOMM 2006), Barcelona, Spain, April 2006. IEEE Computer Society.

[23] Domenico Talia and Paolo Trunfi o. Toward a Synergy Between P2P and Grids. IEEE Internet Computing, 7(4):94-96, 2003.

[24] Nicolas Théodoloz. DHT-based Routing and Discovery in JXTA. Master's thesis, School of Computer and Communication Sciences, February 2004. 
[25] Bernard Traversat, Mohamed Abdelaziz, Dave Doolin, Mike Duigou, Jean-Christophe Hugly, and Eric Pouyoul. Project JXTA-C: Enabling a Web of Things. In 36th Annual Hawaii International Conference on System Sciences (HICSS '03), page 282b, Big Island, Hawaii, January 2003. IEEE Computer Society.

[26] Bernard Traversat, Mohamed Abdelaziz, and Eric Pouyoul. Project JXTA: A LooselyConsistent DHT Rendezvous Walker. http://www. jxta.org/docs/jxta-dht.pdf, March 2003.

[27] Bernard Traversat, Ahkil Arora, Mohamed Abdelaziz, Mike Duigou, Carl Haywood, JeanChristophe Hugly, Eric Pouyoul, and Bill Yeager. Project JXTA 2.0 Super-Peer Virtual Network. http://www. jxta.org/project/www/docs/JXTA2.0protocols1.pdf, May 2003.

[28] Bamboo. http://bamboo-dht.org/.

[29] FreePastry. http://freepastry.rice.edu/.

[30] JXTA Company Spotlight. http://www.jxta.org/companies/ companyarchive.html.

[31] JXTA University Spotlight. http://www.jxta.org/universities/ universityarchive.html.

[32] JXTA specifi cation project. http://spec.jxta.org/.

$\operatorname{RR} \mathrm{n}^{\circ} 1$ 


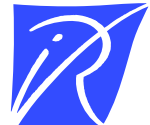

Unité de recherche INRIA Rennes

IRISA, Campus universitaire de Beaulieu - 35042 Rennes Cedex (France)

Unité de recherche INRIA Futurs : Parc Club Orsay Université - ZAC des Vignes 4, rue Jacques Monod - 91893 ORSAY Cedex (France)

Unité de recherche INRIA Lorraine : LORIA, Technopôle de Nancy-Brabois - Campus scientifi que

615, rue du Jardin Botanique - BP 101 - 54602 Villers-lès-Nancy Cedex (France)

Unité de recherche INRIA Rhône-Alpes : 655, avenue de l'Europe - 38334 Montbonnot Saint-Ismier (France)

Unité de recherche INRIA Rocquencourt : Domaine de Voluceau - Rocquencourt - BP 105 - 78153 Le Chesnay Cedex (France)

Unité de recherche INRIA Sophia Antipolis : 2004, route des Lucioles - BP 93 - 06902 Sophia Antipolis Cedex (France)

INRIA - Domaine de Voluceau - Rocquencourt, BP 105 - 78153 Le Chesnay Cedex (France) 